\title{
LUMINESCENCE AND ENERGY MIGRATION IN A TWO-DIMENSIONAL SYSTEM: $\mathrm{NaEuTiO}_{4}$
}

\author{
P.A.M. BERDOWSKI and G. BLASSE \\ Solid State Department, Physics laboratory, Utrecht University, P.O. Box 80.000 , \\ 3508 TA Utrechi, The Netherlands
}

Received 5 January 1984

\begin{abstract}
Energy transfer processes in compounds $\mathrm{NaGd}_{1-x} \mathrm{Eu}_{x} \mathrm{TiO}_{4}(0<x \leqslant 1)$ have been evaluated. It is shown that in these compounds energy migration to quenching centres occurs down to the lowest temperatures if $x>0.3$. Transfer from intrinsic $\mathrm{Eu}^{3+}$ ions to extrinsic $\mathrm{Eu}^{3+}$ ions has been observed. The nature of quenching centres and trap centres has been clarified. The characteristics of the energy migration processes can be explained using a two-dimensional diffusion model. The temperature dependence of the hopping rate can be explained assuming phonon-assisted energy transfer.
\end{abstract}

\section{Introduction}

In the last decade the luminescence of concentrated rare-earth systems has been studied extensively. Especially the luminescence of neodymium compounds in which concentration quenching is ineffective has been a subject of research because of their possible application as minilasers.

Recently Kellendonk and Blasse reported on the luminescence and energy transfer characteristics in $\mathrm{EuAl}_{3} \mathrm{~B}_{4} \mathrm{O}_{12}$ (ref. [1]). They observed migration of the excitation energy through the $\mathrm{Eu}^{3+}$ sublattice above $60 \mathrm{~K}$. However, no migration could be detected at lower temperature. This was ascribed to the fact that at low temperatures only the ${ }^{7} \mathrm{~F}_{0}$ ground state is populated. The process of energy migration within the $\mathrm{Eu}^{3+}$ system involves the decay of one ion from an energy level $i$ to $f$ accompanied by the simultaneous excitation of a nearby ion from $i^{\prime}$ to $f^{\prime}$. The theory for the energy transfer process was initially developed by Förster [2] and later extended by Dexter [3]. For multipolar coupling the probability of energy transfer depends on the probability of the multipolar transitions on the ions involved ( $i$ to $f$ and $i^{\prime}$ to $f^{\prime}$ ). For $\mathrm{Eu}^{3+}$ at low temperatures the only resonant transition giving rise to energy transfer is ${ }^{5} D_{0} \leftrightharpoons{ }^{7} F_{0}$. This transition is strongly forbidden which makes transfer at low temperatures very improbable. As the temperature is increased, higher-lying

0022-2313/84/\$03.00 @ Elsevier Science Publishers B.V.

(North-Holland Physics Publishing Division) 
${ }^{7} \mathrm{~F}_{J}$ levels are thermally populated and additional resonant transition pairs become possible $\left({ }^{5} \mathrm{D}_{0} \leftrightharpoons{ }^{7} \mathrm{~F}_{1} ;{ }^{5} \mathrm{D}_{0} \leftrightharpoons{ }^{7} \mathrm{~F}_{2}\right)$.

It is known that in some compounds the ${ }^{5} \mathrm{D}_{0} \leftrightharpoons{ }^{7} \mathrm{~F}_{0}$ transition is not strictly forbidden $[4,7]$. This can be explained by the presence of a linear crystal field at the rare-earth site in these compounds [8]. The aim of the present investigations is to study the effect of a linear crystal field at the $\mathrm{Eu}^{3+}$ site on the energy migration within the $\mathrm{Eu}^{3+}$ sublattice. Especially the transfer characteristics at low temperatures are interesting, because the ${ }^{5} \mathrm{D}_{0} \leftrightharpoons{ }^{7} \mathrm{~F}_{0}$ transition is no longer strictly forbidden.

We investigated the luminescence properties of powder samples of $\mathrm{NaGd}_{1-x} \mathrm{Eu}_{x} \mathrm{TiO}_{4}(0<x \leqslant 1)$ using laser site-selection and time-resolved spectroscopy. An idealized crystal structure of the sodium lanthanide titanates has been proposed by Blasse [9]. This structure is presented in fig. 1. According to this description these compounds have the $\mathrm{Sr}_{2} \mathrm{TiO}_{4}$ (or $\mathrm{K}_{2} \mathrm{NiF}_{4}$ ) structure with long-range order between the $\mathrm{Na}^{+}$and $\mathrm{Ln}^{3+}$ ions on the $\mathrm{Sr}^{2+}$ sites. The luminescence properties of some $\mathrm{Eu}^{3+}$-activated lanthanide titanates were described in [10]. The results of these investigations could be explained by the proposed crystal structure. For two reasons these compounds seemed to be interesting hosts for studying migration among $\mathrm{Eu}^{3+}$ ions:

(a) With the proposed crystal structure they contain only one crystallographic lanthanide site with a symmetry $\left(\mathrm{C}_{4 \mathrm{v}}\right)$ which lifts the ${ }^{7} \mathrm{~F}_{0} \leftrightharpoons{ }^{5} \mathrm{D}_{0}$ selection rule;

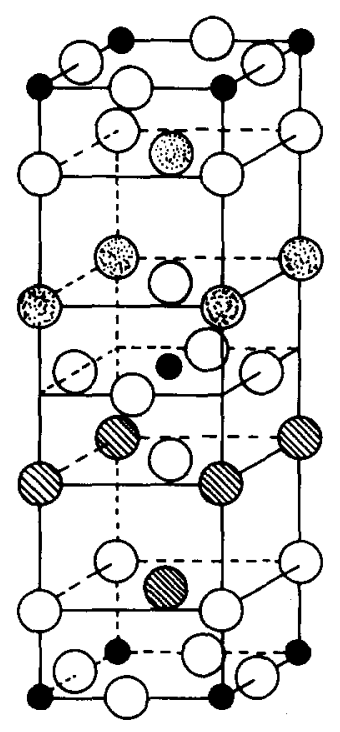

Fig. 1. Idealized crystal structure of $\mathrm{NaEuTiO}_{4}$. Black circles: $\mathrm{Ti}^{4+}$; open circles $\mathrm{O}^{2-}$; circles hatched with lines: $\mathrm{Na}^{+}$; circles hatched with dots: $\mathrm{Eu}^{3+}$. 
(b) The shortest Eu-Eu distance is rather short, viz. $3.7 \AA$ A.

With laser site-selection and time-resolution techniques it was possible to monitor in a powder of $\mathrm{NaEuTiO}_{4}$ energy migration among the intrinsic $\mathrm{Eu}^{3+}$ ions to extrinsic $\mathrm{Eu}^{3+}$ ions down to the lowest temperatures $(1.2 \mathrm{~K})$. This migration is also evident from the concentration quenching which occurred at all temperatures. By analyzing the decay curves we obtained values for the diffusion constant of the energy migration within the $\mathrm{Eu}^{3+}$ system.

\section{Experimental}

All measurements described in this article were performed on powdered samples. The compounds were prepared by firing intimate mixtures of $\mathrm{Na}_{2} \mathrm{CO}_{3}$ (anhydrous), $\mathrm{Eu}_{2} \mathrm{O}_{3}, \mathrm{Gd}_{2} \mathrm{O}_{3}$ and $\mathrm{TiO}_{2}$ at $1050^{\circ} \mathrm{C}$ in dry air. An excess amount of $\mathrm{Na}_{2} \mathrm{CO}_{3}$ ( $\left.7.5 \mathrm{wt} \%\right)$ was needed to compensate for evaporation losses. All samples were checked by $\mathrm{X}$-ray powder diffraction using $\mathrm{CuK} \alpha$ radiation.

For the low-temperature measurements the samples were immersed into liquid helium in a Thor bath cryostat (S-100). The temperature of the samples could be regulated between $1.2 \mathrm{~K}$ and room temperature using a temperature controller (Thor cryogenics models 3020 II). The temperature was measured using a $\mathrm{Fe} / \mathrm{Rh}$ resistance thermometer.

For the performance of the emission spectra a tunable dye laser (Molectron DL 200) pumped with a nitrogen laser (Molectron UV 14) was used as an excitation source. The laser generated a pulse with a peak power of $30 \mathrm{~kW}$ and a width of about $10 \mathrm{~ns}$. For all measurements the laser was set at a fixed repetition rate of about $40 \mathrm{~Hz}$. The resolution of this equipment amounts to $0.03 \mathrm{~nm}$ at $600 \mathrm{~nm}$ (about $1 \mathrm{~cm}^{-1}$ ). The emission of the laser-excited samples was focused on the entrance slit of a Spex 1704X high-resolution monochromator and detected by a cooled photomultiplier (RCA type C31034). To record the time-resolved spectra a PAR model 162/165 boxcar averager was used. A Philips PM 2436 multimeter was used for time averaging and amplification of the photomultiplier signal in order to record time-integrated emission and excitation spectra. Decay curves were recorded using the above-mentioned photomultiplier and an ORTEC photon-counting system (ORTEC 9301 fast preamplifier, ORTEC 574 fast timing amplifier and the ORTEC $436100 \mathrm{MHz}$ discriminator) in combination with a fast Nicolet model 1170 multichannel analyzer. Intensity-versus-time data could be recorded and processed in 1024 channels with a maximum resolution of $1 \mu$ s per channel. The intensity measurements were performed on a Perkin-Elmer spectrofluorometer (MPF3L) equipped with an Oxford Instruments CF 100 liquid helium flow cryostat and Hamamatsu R 928 photomultipliers (spectral resolution $\sim 1 \mathrm{~nm}$ ). 


\section{Results}

\subsection{Spectral properties}

The excitation spectrum of the $\mathrm{Eu}^{3+}$ emission of $\mathrm{NaEuTiO}_{4}$ consists of a broad band with a maximum at $310 \mathrm{~nm}$ and lines in the region 350 to $600 \mathrm{~nm}$. For lower concentrations of $\mathrm{Eu}^{3+}$ the band shifts to shorter wavelengths. This effect has already been described in [10]. At low Eu ${ }^{3+}$ concentrations $(x \leqslant 0.1)$ the band corresponds to the absorption of the exciting radiation by the $\mathrm{TiO}_{6}$ groups followed by energy transfer to $\mathrm{Eu}^{3+}$. At higher concentrations the charge-transfer band of the $\mathrm{Eu}^{3+}$ ion dominates. The lines in the region 350 to $600 \mathrm{~nm}$ correspond to transitions within the $4 \mathrm{f}^{6}$ configuration of Eu ${ }^{3+}$.

Figure 2 shows the emission spectrum of $\mathrm{NaEuTiO}_{4}$ recorded at $4.2 \mathrm{~K}$ under high resolution. A dye laser was used as an excitation source and the excitation wavelength was $396.8 \mathrm{~nm}$. As shown by Linarès and Blanchard [11] for the emission spectra of $\mathrm{NaLnTiO}_{4}: \mathrm{Eu}(\mathrm{Ln}=\mathrm{Gd}, \mathrm{Y}$ or $\mathrm{Lu})$, more transitions can be observed in the emission spectrum of $\mathrm{NaEuTiO}_{4}$ recorded with high resolution than reported in [10]. Apparently the site symmetry of the intrinsic $\mathrm{Eu}^{3+}$ ions is lower than $\mathrm{C}_{4 \mathrm{v}}$. This is probably related to the fact that the $\mathrm{Ti}^{4+}$ ion is off-centre, which can be concluded from the infrared and Raman spectra [12]. A structure refinement has to be performed to elucidate this effect. The emission spectrum consists of transitions from the ${ }^{5} \mathrm{D}_{0}$ level to the ${ }^{7} \mathrm{~F}_{J}$ manifold. The emission from higher ${ }^{5} \mathrm{D}$ states could only be observed for samples containing concentrations of $\mathrm{Eu}^{3+}$ ions with $x<0.3$. The absence of

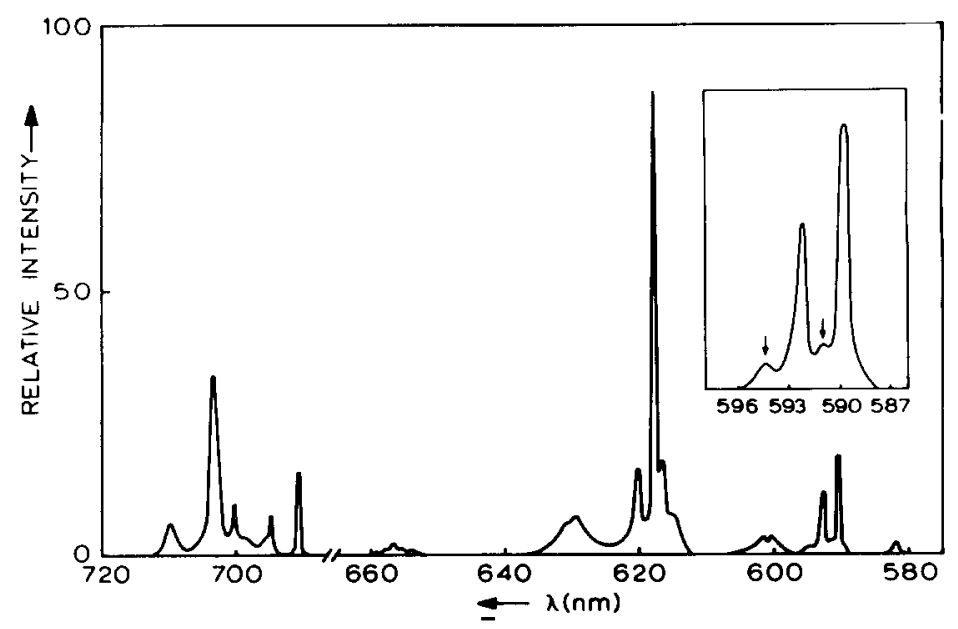

Fig. 2. Emission spectrum of $\mathrm{NaEuTiO}_{4}$, recorded at $4.2 \mathrm{~K}$ (excitation wavelength $396.8 \mathrm{~nm}$ ). The insert gives the part of the emission spectrum around $590 \mathrm{~nm}$. The arrows indicate the satellite lines. 
${ }^{5} \mathrm{D}_{1}$ and ${ }^{5} \mathrm{D}_{2}$ lines in the $\mathrm{Eu}^{3+}$ emission of $\mathrm{NaEuTiO}_{4}$ is due to cross relaxation.

In addition to the absence of ${ }^{5} \mathrm{D}_{1}$ and ${ }^{5} \mathrm{D}_{2}$ lines in the emission spectrum of $\mathrm{NaEuTiO}_{4}$ there is another difference in comparison with the emission spectrum of $\mathrm{NaGd}_{0.99} \mathrm{Eu}_{0.01} \mathrm{TiO}_{4}$. In the emission spectrum of $\mathrm{NaEuTiO}_{4}$ we can clearly distinguish weak lines in the vicinity of most of the main lines (e.g. those near 589.7 and $592.6 \mathrm{~nm}$ ). These lines could be detected in the spectra of the samples with high $\mathrm{Eu}^{3+}$ concentrations. The presence of these so-called "satellite" lines indicates that there are different types of $\mathrm{Eu}^{3+}$ ions.

In order to obtain information on the types of $\mathrm{Eu}^{3+}$ ions we recorded the excitation spectra for the ${ }^{5} \mathrm{D}_{0} \rightarrow{ }^{7} \mathrm{~F}_{2}$ main emission line $(616.9 \mathrm{~nm})$ and a satellite line $(615.9 \mathrm{~nm})$ of $\mathrm{NaGd}_{0.99} \mathrm{Eu}_{0.01} \mathrm{TiO}_{4}$ in the ${ }^{7} \mathrm{~F}_{0} \rightarrow{ }^{5} \mathrm{D}_{0}$ spectral range. The results are shown in fig. 3. The excitation spectrum of the main line (fig. 3a) consists of one sharp line at $580.75 \mathrm{~nm}$ without any observable satellite lines. The linewidth of this transition is $2.4 \mathrm{~cm}^{-1}$. The excitation spectrum of the satellite line at $615.9 \mathrm{~nm}$ shows the same main line, but also at least three satellite lines (see fig. 3b). This suggests the same number of different $\mathrm{Eu}^{3+}$

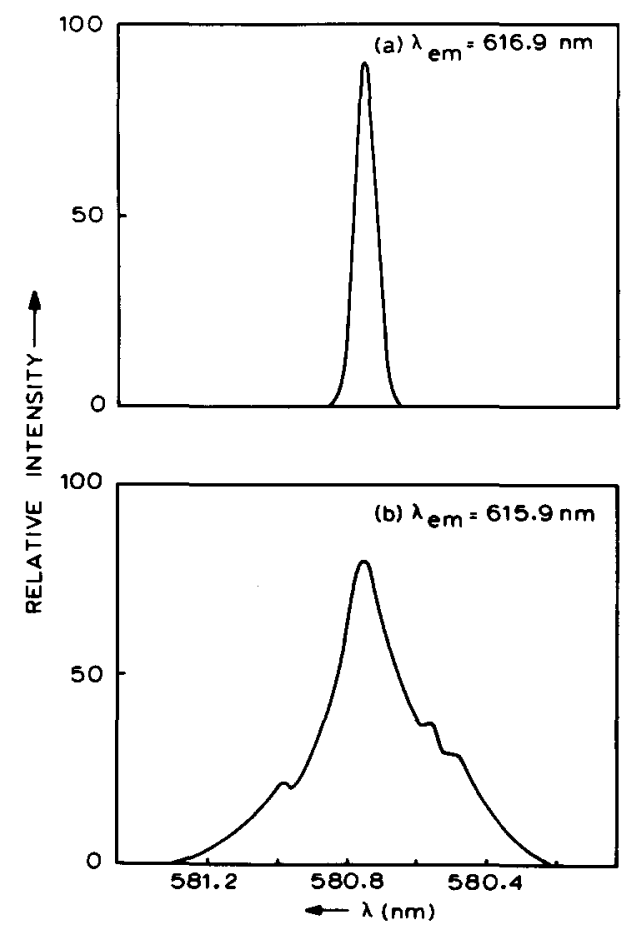

Fig. 3. Excitation spectra of the emission of $\mathrm{NaGd}_{0.99} \mathrm{Eu}_{0.01} \mathrm{TiO}_{4}$, recorded at $4.2 \mathrm{~K}$ in the ${ }^{7} \mathrm{~F}_{0} \rightarrow{ }^{5} \mathrm{D}_{0}$ spectral region. (a) Emission wavelength $616.9 \mathrm{~nm}$; (b) Emission wavelength $615.9 \mathrm{~nm}$. 
ions. The broadening of the lines is due to a lower resolution of the optical equipment because of the low intensity of the emission at $615.9 \mathrm{~nm}$. The main line is also monitored in the latter case because of the large slit width and the coincidence of a main line with the satellite line (see below). When the same excitation spectra were recorded for $\mathrm{NaEuTiO}_{4}$, even the excitation spectrum of the $616.9 \mathrm{~nm}$ main line showed satellite lines in the vicinity of the main excitation peak (see fig. 4a). The main peak has the same position as for $\mathrm{NaGd}_{0.99} \mathrm{Eu}_{0.01} \mathrm{TiO}_{4}$. The linewidth is $5.2 \mathrm{~cm}^{-1}$. The excitation spectrum of the satellite line at $615.9 \mathrm{~nm}$ shows a main line at higher wavelength, and the excitation line of the intrinsic emission. Figures $3 \mathrm{~b}$ and $4 \mathrm{~b}$ show that there is a difference in relative intensity between the satellite peaks and the intrinsic peak in $\mathrm{NaGd}_{0.99} \mathrm{Eu}_{0.01} \mathrm{TiO}_{4}$ and in $\mathrm{NaEuTiO}_{4}$. This is probably due to concentration quenching of the intrinsic emission (see below).

We used a tunable dye laser to excite selectively the intrinsic $\mathrm{Eu}^{3+}$ ion with the ${ }^{7} \mathrm{~F}_{0} \leftrightharpoons{ }^{5} \mathrm{D}_{0}$ line at $580.75 \mathrm{~nm}$ and the extrinsic $\mathrm{Eu}^{3+}$ ion with the $580.95 \mathrm{~nm}$ line. Figure 5 shows parts of the ${ }^{5} \mathrm{D}_{0} \rightarrow{ }^{7} \mathrm{~F}_{2}$ emission spectra of the two Eu ${ }^{3+}$ centres. These overlap strongly which explains the observations mentioned

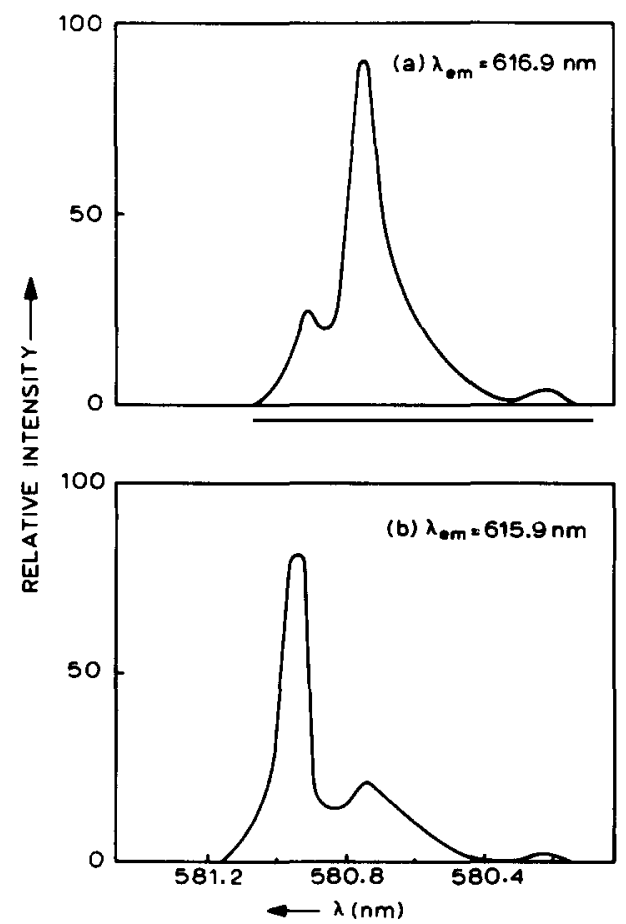

Fig. 4. Excitation spectra of the emission of $\mathrm{NaEuTiO}_{4}$, recorded at $4.2 \mathrm{~K}$, in the ${ }^{7} \mathrm{~F}_{0} \rightarrow{ }^{5} \mathrm{D}_{0}$ spectral region. (a) Emission wavelength $616.9 \mathrm{~nm}$; (b) Emission wavelength $615.9 \mathrm{~nm}$. 


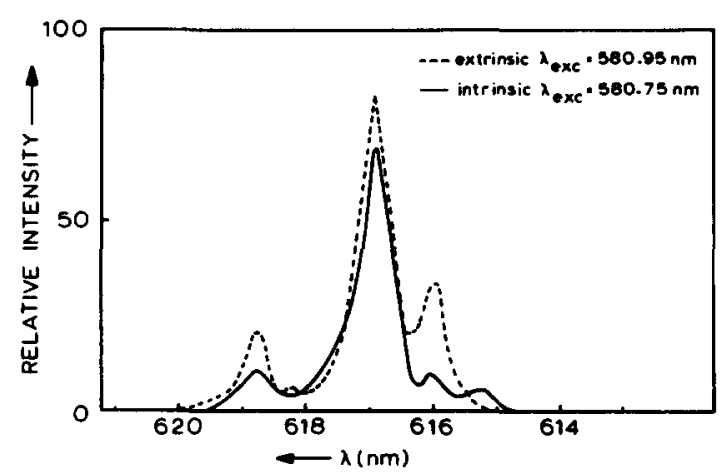

Fig. 5. ${ }^{5} \mathrm{D}_{0} \rightarrow{ }^{7} \mathrm{~F}_{2}$ emission spectra of different $\mathrm{Eu}^{3+}$ ions in $\mathrm{NaEuTiO}_{4}$ upon narrow line excitation, $T=4.2 \mathrm{~K}$. Continuous line: spectrum of the intrinsic $\mathrm{Eu}^{3+}$ ions (excitation wavelength $580.75 \mathrm{~nm}$ ). Dashed line: spectrum of the extrinsic $\mathrm{Eu}^{3+}$ ions (excitation wavelength $580.95 \mathrm{~nm}$ ).

above. The relative intensities and the position of the various lines are different for the two types of $\mathrm{Eu}^{3+}$ ions.

Figures 6 and 7 show the excitation spectra of the emission of the intrinsic

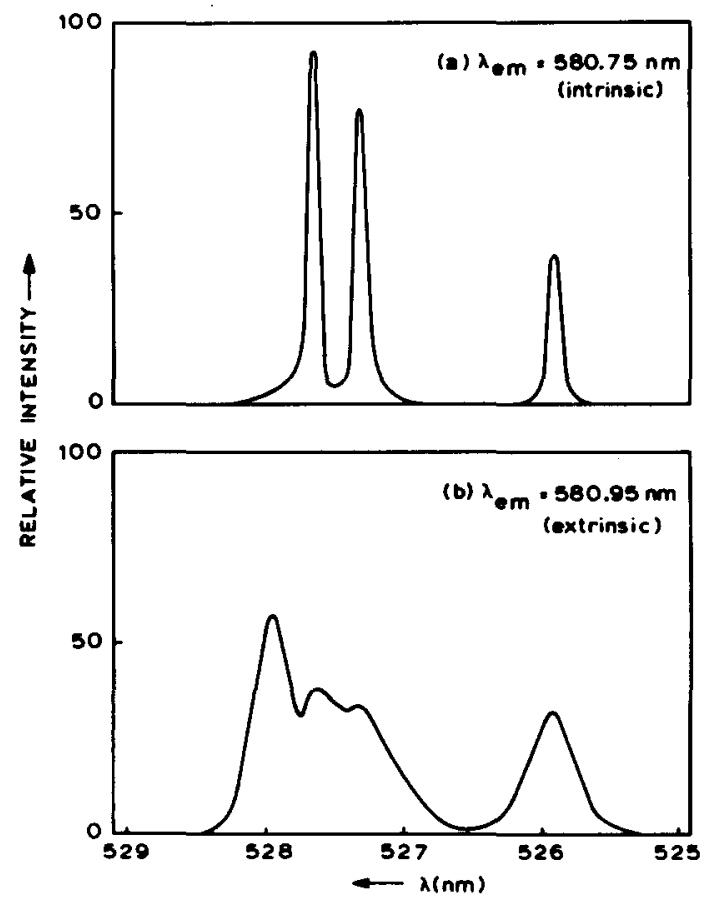

Fig. 6. Excitation spectra of the emission of $\mathrm{NaEuTiO}_{4}$ in the ${ }^{7} \mathrm{~F}_{0} \rightarrow{ }^{5} \mathrm{D}_{1}$ spectral region, at $4.2 \mathrm{~K}$. (a) Emission wavelength $580.75 \mathrm{~nm}$; (b) Emission wavelength $580.95 \mathrm{~nm}$. 

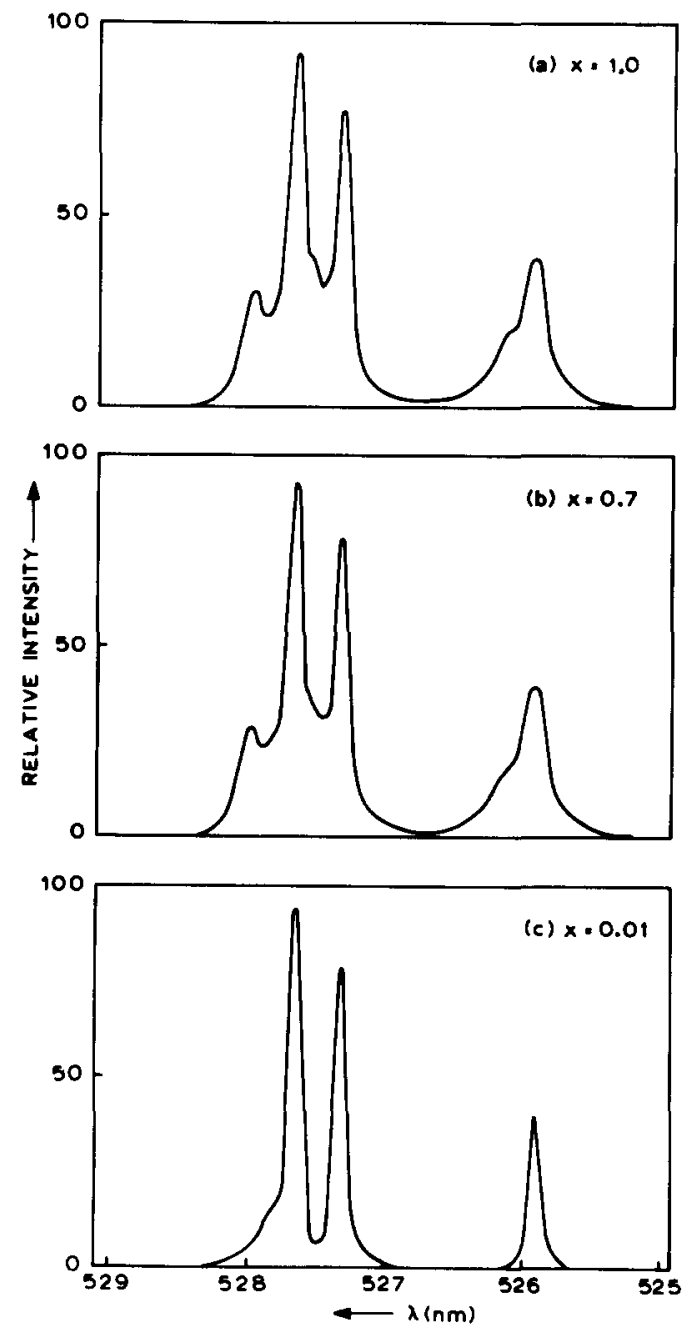

Fig. 7. Excitation spectra of the emission of $\mathrm{NaGd}_{1-x} \mathrm{Eu}_{x} \mathrm{TiO}_{4}$ in the ${ }^{7} \mathrm{~F}_{0} \rightarrow{ }^{5} \mathrm{D}_{1}$ spectral region, $T=4.2 \mathrm{~K}$, as a function of $x$. The emission wavelength is $616.9 \mathrm{~nm}$.

and the extrinsic Eu ${ }^{3+}$ ions in $\mathrm{NaEuTiO}_{4}$ at $4.2 \mathrm{~K}$ in the ${ }^{7} \mathrm{~F}_{0} \rightarrow{ }^{5} \mathrm{D}_{1}$ spectral region. Figure 7a shows this excitation spectrum for emission wavelength 616.9 $\mathrm{nm}$. Figure $6 \mathrm{a}$ represents the excitation spectrum of the intrinsic $\mathrm{Eu}^{3+}$ ions. The number of peaks (3), their positions and relative intensities are in agreement with the results of measurements on $\mathrm{NaGd}_{0.99} \mathrm{Eu}_{0.01} \mathrm{TiO}_{4}$ and with the results of Linarès and Blanchard [11]. Figure $6 \mathrm{~b}$ shows the excitation spectrum of the extrinsic $\mathrm{Eu}^{3+}$ ion. The number of peaks is not easy to 
determine, but seems to be 4 . The intrinsic $\mathrm{Eu}^{3+}$ ion peaks at 527.3 and 527.7 $\mathrm{nm}$ can be recognized in this spectrum. Their presence, if not due to the larger slit width, indicates only a small amount of energy transfer from the intrinsic $\mathrm{Eu}^{3+}$ ions to the extrinsic $\mathrm{Eu}^{3+}$ ions.

In order to determine the concentration of extrinsic $\mathrm{Eu}^{3+}$ ions with ${ }^{7} \mathrm{~F}_{0} \rightarrow{ }^{5} \mathrm{D}_{0}$ excitation line at $580.95 \mathrm{~nm}$, we recorded the emission spectra of $\mathrm{NaGd}_{0.99} \mathrm{Eu}_{0.01} \mathrm{TiO}_{4}$ and $\mathrm{NaEuTiO}_{4}$ at $4.2 \mathrm{~K}$ upon site-selective excitation of these ions. If an equal absorption strength is assumed for the ${ }^{7} \mathrm{~F}_{0} \rightarrow{ }^{5} \mathrm{D}_{0}$ transitions of the two different $\mathrm{Eu}^{3+}$ ions, the concentration of extrinsic ions can be estimated from the emission spectra. For $\mathrm{NaGd}_{0.99} \mathrm{Eu}_{0.01} \mathrm{TiO}_{4}$ it was found that about $1 \%$ of the $\mathrm{Eu}^{3+}$ ions was incorporated as extrinsic ions, whereas the concentration of extrinsic $\mathrm{Eu}^{3+}$ ions in $\mathrm{NaEuTiO}_{4}$ was $10 \%$ of the total amount of $\mathrm{Eu}^{3+}$ ions. This discrepancy between the estimates of the concentration must be ascribed to concentration quenching of the intrinsic emission in $\mathrm{NaEuTiO}_{4}$, as will be discussed below. No noticeable difference between the X-ray diffraction and the infrared spectra of both compounds could be observed. The estimate for the diluted system seems to be the more reliable one.

The influence of the $\mathrm{Eu}^{3+}$ concentration in $\mathrm{NaGd}_{1-x} \mathrm{Eu}_{x} \mathrm{TiO}_{4}$ on the relative intensities of the peaks is illustrated in fig. 7 , which shows the excitation spectra of the $616.9 \mathrm{~nm}$ emission line at $4.2 \mathrm{~K}$ for different $\mathrm{Eu}^{3+}$ concentrations. In fig. 7a the excitation spectrum of $\mathrm{NaEuTiO}_{4}$ is shown. The excitation spectrum of $\mathrm{NaGd}_{0.3} \mathrm{Eu}_{0.7} \mathrm{TiO}_{4}$ (fig. 7b) differs hardly from the spectrum of $\mathrm{NaEuTiO}_{4}$. Figure $7 \mathrm{c}$ presents this excitation spectrum in the case of $\mathrm{NaGd}_{0.99} \mathrm{Eu}_{0.01} \mathrm{TiO}_{4}$. This spectrum resembles the ${ }^{7} \mathrm{~F}_{0} \rightarrow{ }^{5} \mathrm{D}_{1}$ excitation spectrum of the intrinsic $\mathrm{Eu}^{3+}$ ions in $\mathrm{NaEuTiO}_{4}$ (see fig. 6b). Only at 527.8 $\mathrm{nm}$ a small satellite line can be observed. The increase of the satellite lines relative to the main lines with increasing value of $x$ is due to concentration quenching of the main line emission (see below).

From fig. 1 it is clear that the lanthanide and the sodium ions form double layers perpendicular to the $c$-axis. The difference in charge between these layers is probably compensated by a shift of the $\mathrm{Ti}^{4+}$ ions towards the $\mathrm{Na}^{+}$ ions. As stated above this is supported by the observed position of the Ti-O stretching band in the infrared and Raman spectrum [12]. Due to this shift the $\mathrm{Ti}^{4+}$ are five instead of six coordinated. It seems not unreasonable to assume that the extrinsic $\mathrm{Eu}^{3+}$ ions may originate from a slight deviation from the perfect superstructure. One extrinsic $\mathrm{Eu}^{3+}$ ion is then an $\mathrm{Eu}^{3+}$ ion in the $\mathrm{Na}^{+}$ layer, another is a $\mathrm{Eu}^{3+}$ ion next to $\mathrm{Na}^{+}$ion in the $\mathrm{Eu}^{3+}$ layer.

\subsection{Energy transfer}

\subsubsection{Concentration and temperature dependence of the luminescence}

A clear indication for energy migration is the observation of concentration 
quenching of the intrinsic emission. Powders with composition $\mathrm{NaGd}_{1-x} \mathrm{Eu}_{x} \mathrm{TiO}_{4}(0<x \leqslant 1)$ were prepared and the time-integrated emission spectrum of each of the samples was recorded in the region between $8 \mathrm{~K}$ and $300 \mathrm{~K}$ (excitation wavelength $397 \mathrm{~nm}$ ). The integrated emission intensity of the ${ }^{5} \mathrm{D}_{0} \rightarrow{ }^{7} \mathrm{~F}_{J}(0 \leqslant J \leqslant 4)$ transitions was determined for each sample.

Figure 8 presents the integrated emission intensities of $\mathrm{NaGd}_{1-x} \mathrm{Eu}_{x} \mathrm{TiO}_{4}$ as a function of $x$ at $8 \mathrm{~K}$ and $300 \mathrm{~K}$. It is clear that concentration quenching is observed at both temperatures. At higher temperatures the emission intensity of samples with $x \geqslant 0.5$ was only a few percent of that of the sample with $x=0.3$. At $8 \mathrm{~K}$ this percentage was about 30 . The sample with $x=0.3$ gives a bright luminescence at room temperature. Figure 9 presents the temperature dependence of the integrated emission intensity of $\mathrm{NaEuTiO}_{4}$ (excitation wavelength $397 \mathrm{~nm}$ ). This figure shows that in the temperature region between $8 \mathrm{~K}$ and $60 \mathrm{~K}$ the emission intensity decreases rapidly. Above $80 \mathrm{~K}$ the intensity reaches a constant value of about $2 \%$ of the maximum value at $8 \mathrm{~K}$. This phenomenon was only observed for samples with europium concentrations of $x \geqslant 0.5$. The emission intensity of samples with lower $\mathrm{Eu}^{3+}$ concentrations decreases slowly with increasing temperature: the intensity at $300 \mathrm{~K}$ is about $50 \%$ of the intensity at $8 \mathrm{~K}$. Concentration quenching can be explained by excitation energy migration among the intrinsic $\mathrm{Eu}^{3+}$ ions to quenching centres. At low temperatures the presence of concentration quenching excludes an important role of the extrinsic $\mathrm{Eu}^{3+}$ ions in trapping the migrating

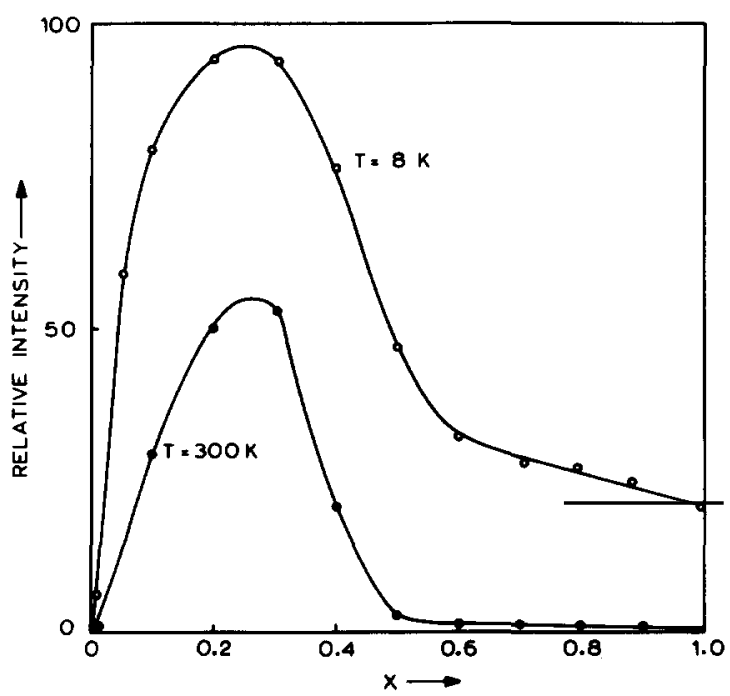

Fig. 8. The relative intensities of the $\mathrm{Eu}^{3+}$ emission of $\mathrm{NaGd}_{1-x} \mathrm{Eu}_{x} \mathrm{TiO}_{4}$ as a function of $x$ at 300 $\mathrm{K}$ and $8 \mathrm{~K}$. 


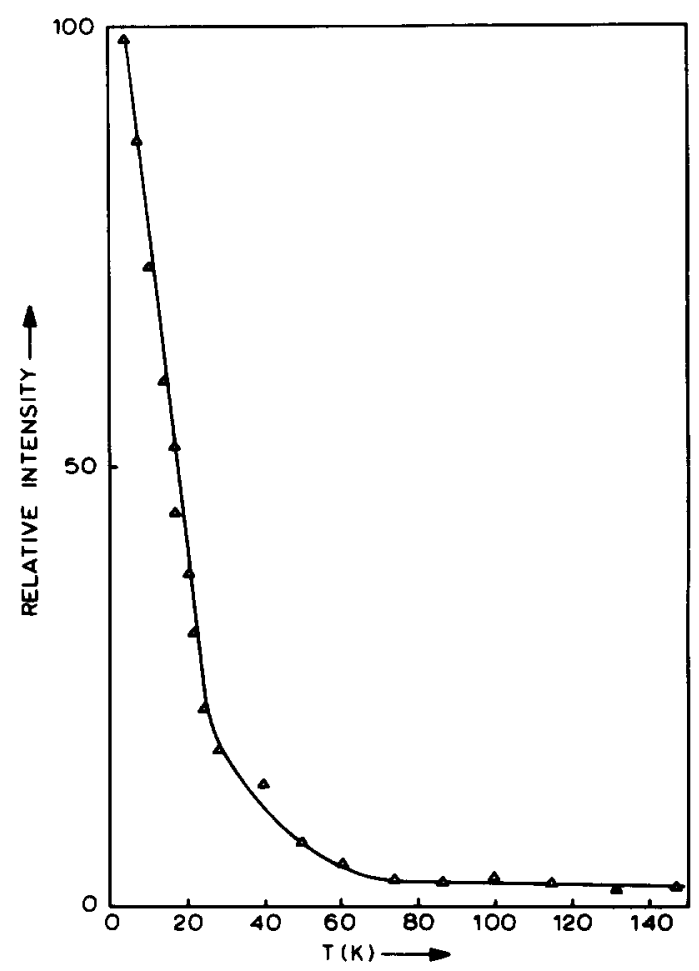

Fig. 9. The temperature dependence of the emission intensity of $\mathrm{NaEuTiO}_{4}$ (excitation wavelength $397 \mathrm{~nm})$.

excitation energy. Also in section 3.1 several spectra point to the predominant role of quenching centres above trap centres. The temperature dependence of the intensity of the luminescence in the concentrated systems can be explained by assuming a temperature dependence of the migration probability, as will be discussed below.

Transition metal ions are potential impurities in titanium oxide. Some of these ions are well-known quenchers of $\mathrm{Eu}^{3+}$ emission. Atomic absorption analysis showed that iron is the main impurity in our samples. Its concentration was found to be relatively high $(\sim 0.1 \mathrm{wt} \%)$.

\subsubsection{Time dependence of the luminescence}

A third experiment to investigate the energy migration in $\mathrm{NaEuTiO}_{4}$ is the measurement of time-resolved spectra of the emission. To monitor any possible energy transfer we measured at $4.2 \mathrm{~K}$ spectra as a function of time in the range $5 \mu \mathrm{s}$ to $2 \mathrm{~ms}$ after the excitation pulse. Figure 10 shows, as an example, the time dependence of the ${ }^{5} \mathrm{D}_{0} \rightarrow{ }^{7} \mathrm{~F}_{2}$ emission upon selective laser excitation of 

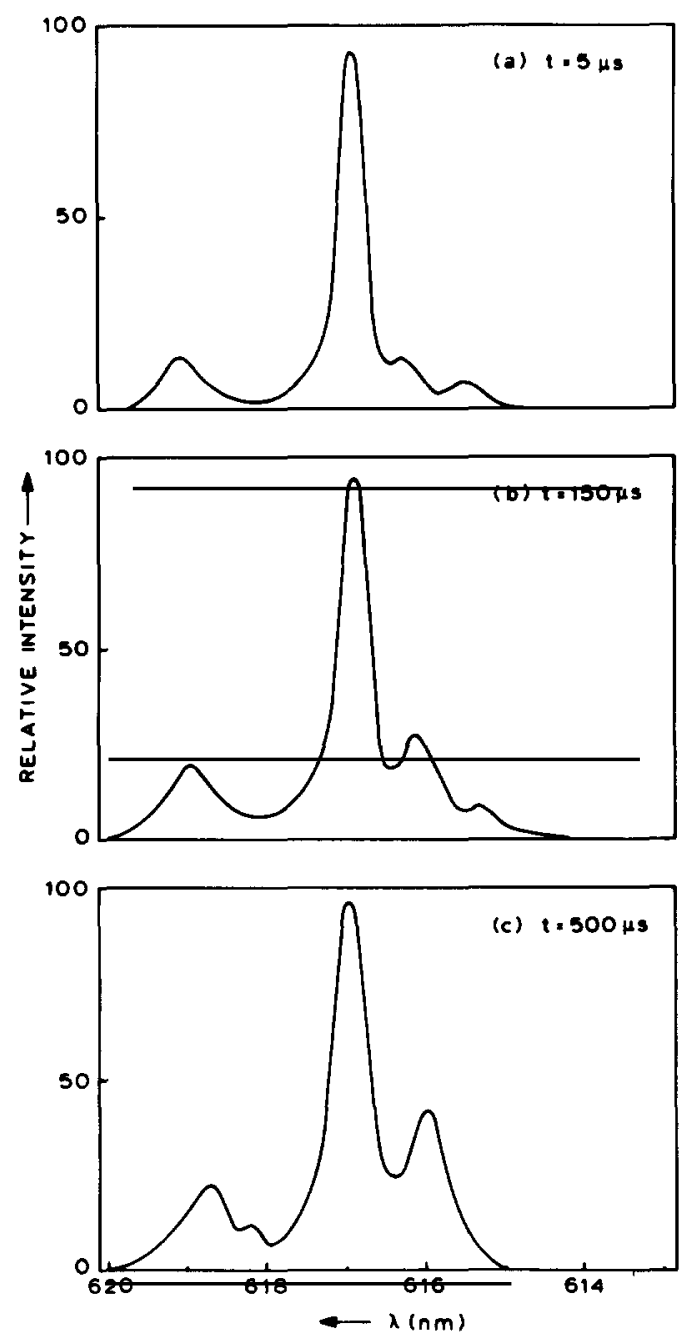

Fig. 10. The time dependence of the emission spectrum after excitation in the intrinsic $\mathrm{Eu}^{3+}$ ions in $\mathrm{NaEuTiO}_{4}$ at $4.2 \mathrm{~K} . t$ indicates the time after the excitation pulse.

the intrinsic $\mathrm{Eu}^{3+}$ ions. The spectrum recorded $5 \mu \mathrm{s}$ after the laser pulse resembles the ${ }^{5} \mathrm{D}_{0} \rightarrow{ }^{7} \mathrm{~F}_{2}$ emission spectrum of the intrinsic $\mathrm{Eu}^{3+}$ ions (see fig. 5). However, with increasing time after the laser pulse, we could observe a buildup of the lines associated with the extrinsic ions at the expense of those of the intrinsic ions. The spectrum recorded $500 \mu \mathrm{s}$ after the pulse had the same shape as the ${ }^{5} \mathrm{D}_{0} \rightarrow{ }^{7} \mathrm{~F}_{2}$ emission spectrum of the extrinsic $\mathrm{Eu}^{3+}$ ions (see fig. 5 ). The integrated emission intensity of this spectrum was only a few percent of 
the intensity of the spectrum recorded $5 \mu \mathrm{s}$ after the pulse. This indicates that most of the intrinsic emission is quenched. It seems reasonable to conclude that energy transfer from the intrinsic $\mathrm{Eu}^{3+}$ ions to the extrinsic $\mathrm{Eu}^{3+}$ ions is responsible for the transient behaviour of the emission in all samples with high $\mathrm{Eu}^{3+}$ concentrations $(x \geqslant 0.6)$. It should be stressed that the concentration quenching experiments show that the greater amount of the excitation energy reaches the quenching centres, and only a small part the extrinsic $\mathrm{Eu}^{3+}$ ions. We also studied the time-resolved spectra after excitation of the extrinsic ions in $\mathrm{NaEuTiO}_{4}$. At low temperatures no energy transfer to intrinsic $\mathrm{Eu}^{3+}$ ions

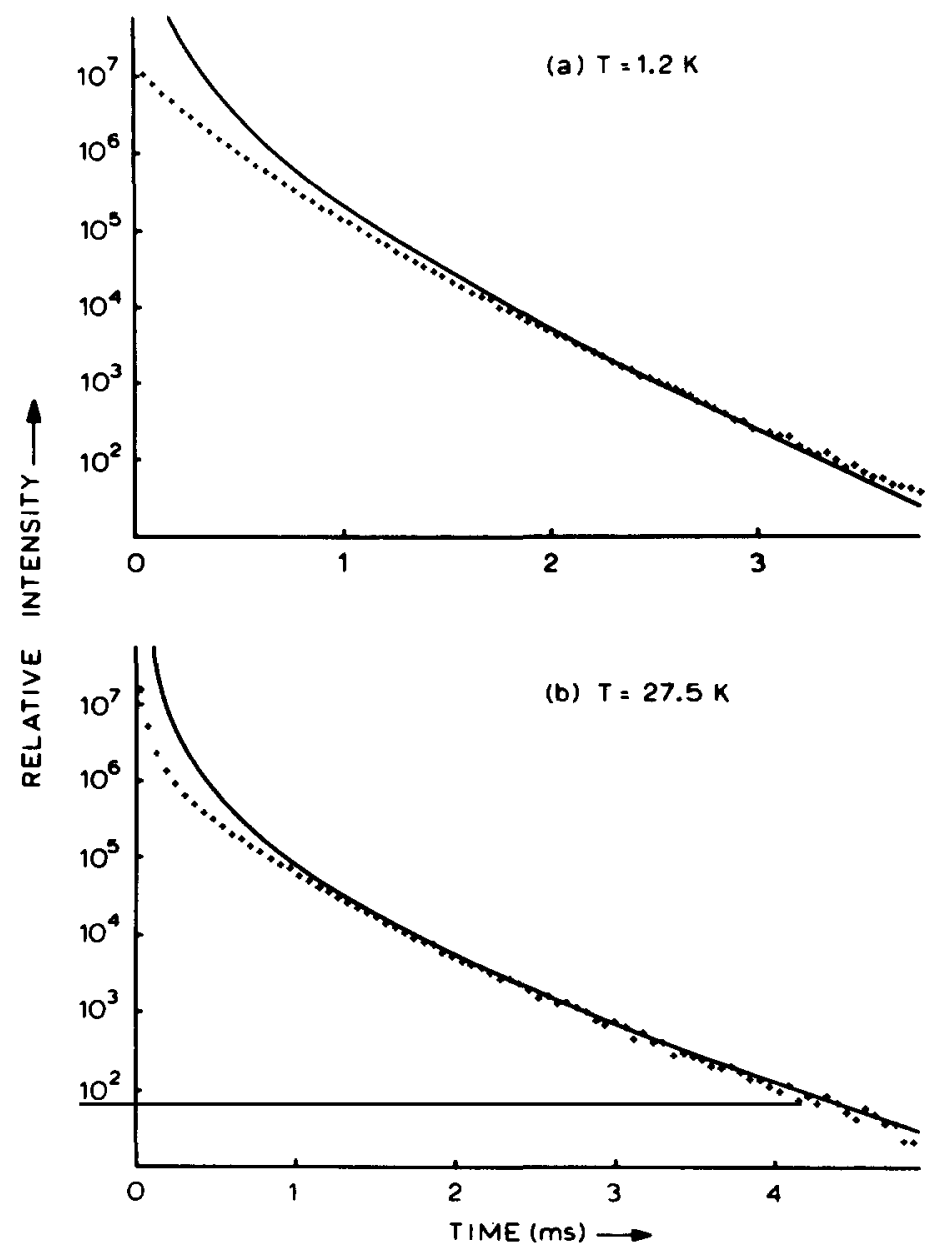

Fig. 11. Decay curves of the intrinsic $\mathrm{Eu}^{3+}$ emission $(616.9 \mathrm{~nm})$ in $\mathrm{NaEuTiO}_{4}$ at $1.2 \mathrm{~K}$ and $27.5 \mathrm{~K}$ (excitation wavelength $580.75 \mathrm{~nm}$ ). 
could be detected. The extrinsic $\mathrm{Eu}^{3+}$ ions seem to trap the excitation energy. At higher temperatures the intensity of the luminescence became too weak to allow any interpretation.

The decay characteristics of the ${ }^{5} \mathrm{D}_{0}$ emission were investigated as a function of temperature both for $\mathrm{NaGd}_{0.99} \mathrm{Eu}_{0.01} \mathrm{TiO}_{4}$ and for $\mathrm{NaEuTiO}_{4}$. For the former the decay curves after excitation in ${ }^{5} \mathrm{D}_{0}$ are exponential in the temperature region under study. The decay time at $1.2 \mathrm{~K}$ was $920 \mu$ s and remained constant up to $300 \mathrm{~K}$. The decay curves of the emission of the intrinsic $\mathrm{Eu}^{3+}$ ions in $\mathrm{NaEuTiO}_{4}$ were recorded for excitation into the intrinsic $\mathrm{Eu}^{3+}$ ions. The decay curves show a deviation from exponential behaviour in the whole temperature region $(1.2-300 \mathrm{~K})$. Even for long times after the laser pulse the decay curves are not exponential. Figure 11 shows the decay curves of the intrinsic emission at $1.2 \mathrm{~K}$ and at $27.5 \mathrm{~K}$. The plotted lines are theoretical fits. As will be discussed below, these curves point to energy migration through the $\mathrm{Eu}^{3+}$ sublattice.

\section{Discussion}

From fig. 8 it follows that the quenching of the luminescence in $\mathrm{NaGd}_{1-x} \mathrm{Eu}_{x} \mathrm{TiO}_{4}$ powders occurs about $x=0.3$ at $300 \mathrm{~K}$. Apparently energy migration among the $\mathrm{Eu}^{3+}$ ions becomes of influence above $x=0.3$. The quenching can be ascribed to loss of migrating excitation energy at a quenching centre. We approximated the quencher concentration by the value of the total iron concentration found by the analysis, viz. 0.5 at $\%$.

The time development of the intrinsic luminescence following pulsed excitation can be used to distinguish between several cases of transfer. Provided the lifetime of the excited state is known, information about transfer between intrinsic ions and between intrinsic and extrinsic ions can be obtained by fitting the intrinsic decay curve to appropriate theoretical expressions. These expressions are described extensively in refs. [13-18]. As stated before the lanthanide ions form double layers perpendicular to the $c$-axis. The distance between these layers $(\sim 10 \AA)$ is considerably larger than the distance between two lanthanide ions in the layers $(\sim 3.7 \AA)$. Because of the reciprocal dependence on $R$ in the case of multipole-multipole interaction $\left(R^{-6} ; R^{-8} ; R^{-10}\right)$ and the exponential dependence on $R(\exp (-R / L))$ in the case of exchange interaction, the interaction strengths between ions in different layers must be orders of magnitude smaller than between nearest neighbours.

This implies that migration among $\mathrm{Eu}^{3+}$ ions occurs in the double layers. For this reason we tried to fit the experimental results to a two-dimensional diffusion model. In this case the intrinsic decay will be non-exponential for all times after the excitation pulse. Neglecting back transfer from trap or killer ions to the intrinsic ions the equation to describe the integrated intensity at 
time $t$ after the excitation pulse has the asymptotic limit for $t \rightarrow \infty$ (ref. [13]):

$I(t)=I(0) \exp \left(-p_{\mathrm{r}} t\right)\left(4 \pi c_{a} a^{-2} D t\right)^{-1}$,

where $I(0)$ is the integrated intensity at $t=0, p_{\mathrm{r}}$ is the radiative decay probability, $c_{a}$ is the total concentration of traps and killers, $a$ is the lattice constant and $D$ the diffusion constant. This equation is only valid for the limit $c_{a} \rightarrow 0$. Although the total concentration of traps and killers is relatively high in $\mathrm{NaEuTiO}_{4}$, we used eq. (1) as an approximation. Figure 11a shows the decay curve of the intrinsic emission at $1.2 \mathrm{~K}$ and the two-dimensional diffusion fit. For the radiative rate we have used $p_{\mathrm{r}}=1090 \mathrm{~s}^{-1}$, the decay rate for the isolated $\mathrm{Eu}^{3+}$ ions in $\mathrm{NaGd}_{0.99} \mathrm{Eu}_{0.01} \mathrm{TiO}_{4}$. The value of $c_{a}$ was taken as $5.5 \times 10^{19} \mathrm{~cm}^{-3}$ and that of $a=3.7$ A. From fig. 8 it is obvious that most of the migrating excitation energy is lost at killer sites. For this reason $c_{a}$ is approximately constant with varying temperature. Figure $11 \mathrm{~b}$ presents the decay curve at $27.5 \mathrm{~K}$. The solid line represents the best fit using the two-dimensional diffusion model. $P_{\mathrm{r}}, c_{a}$ and $a$ were given the same values as for fig. 11a.

As has already been stated, eq. (1) is only valid for long times after the excitation pulse. From figs. $11 \mathrm{a}$ and $11 \mathrm{~b}$ it is obvious that the two-dimensional diffusion fit gives reasonable result in the expected region. All decay curves recorded at various temperatures in the region between $1.2 \mathrm{~K}$ and $300 \mathrm{~K}$ could be fitted using a two-dimensional diffusion model. We conclude therefore that we are dealing with two-dimensional diffusion-limited energy migration.

From the fits on the decay curves it was possible to calculate the diffusion constant $D$. For the decay at $1.2 \mathrm{~K}$ we obtain $D=8 \times 10^{-11} \mathrm{~cm}^{2} \mathrm{~s}^{-1}$. For the decay at $27.5 \mathrm{~K}, D=3 \times 10^{-9} \mathrm{~cm}^{2} \mathrm{~s}^{-1}$. At $300 \mathrm{~K}, D=2 \times 10^{-8} \mathrm{~cm}^{2} \mathrm{~s}^{-1}$. A comparison with the room temperature values of $D=8 \times 10^{-10} \mathrm{~cm}^{2} \mathrm{~s}^{-1}$ found for $\mathrm{EuAl}_{3} \mathrm{~B}_{4} \mathrm{O}_{12}$ (ref. [1]) and $D=1.2 \times 10^{-9} \mathrm{~cm}^{2} \mathrm{~s}^{-1}$ found for $\mathrm{Tb}_{3} \mathrm{Al}_{5} \mathrm{O}_{12}$ (ref. [19]) shows that our $D$ value at $300 \mathrm{~K}$ is relatively high. This could be due to the fact that the nearest neighbour distance in $\mathrm{NaEuTiO}_{4}$ is relatively short.

From the diffusion constant we can calculate the hopping time $\left(t_{\mathrm{H}}\right)$ according to ref. [20]:

$t_{\mathrm{H}}=a^{2} / 4 D$.

The number of steps $(n)$ given by [20]

$n=\tau_{\mathrm{r}} / \tau_{\mathrm{H}}$

is 200 at $1.2 \mathrm{~K}, 8 \times 10^{3}$ at $27.5 \mathrm{~K}$ and $5 \times 10^{4}$ at $300 \mathrm{~K}$.

Figure 12 shows the temperature dependence of the hopping rate $\left(1 / t_{\mathrm{H}} \equiv\right.$ $K_{\mathrm{H}}$ ) among intrinsic $\mathrm{Eu}^{3+}$ ions in $\mathrm{NaEuTiO}_{4}$. The hopping rate increases linearly with temperature in the region $1.2-20 \mathrm{~K}$. From $20 \mathrm{~K}$ up to $35 \mathrm{~K}$ it increases very rapidly. At higher temperatures the hopping rate becomes approximately constant. 


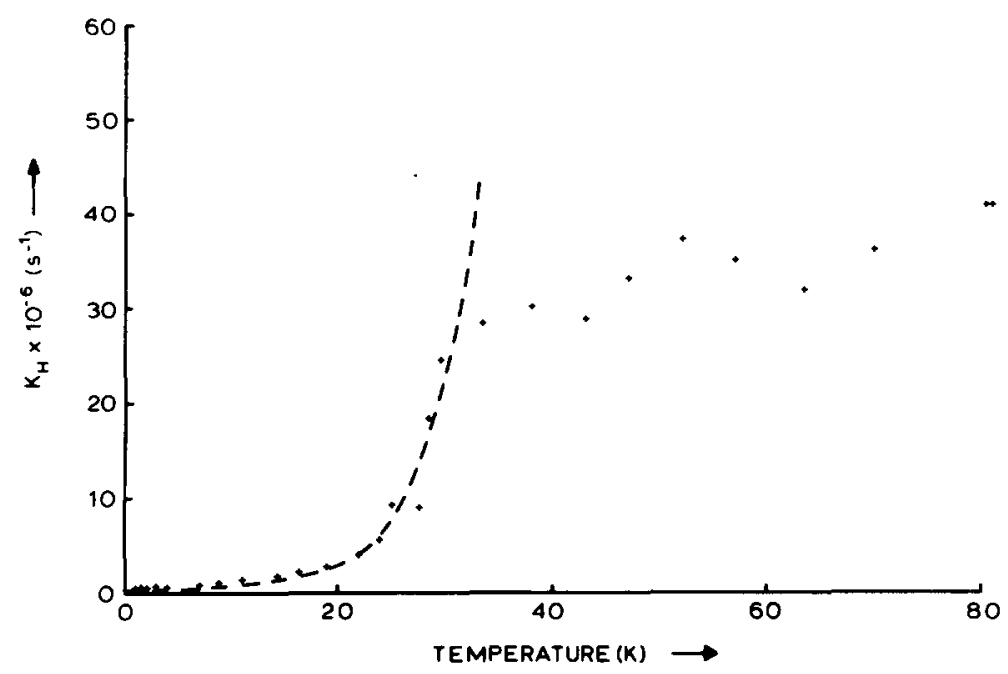

Fig. 12. The temperature dependence of the hopping rate within the intrinsic $\mathrm{Eu}^{3+}$ system in $\mathrm{NaEuTiO}_{4}$. See text for explanation of the theoretical line.

Kellendonk and Blasse [1] explained the temperature dependence of the diffusion constant by thermal population of the ${ }^{7} F_{1}$ and ${ }^{7} F_{2}$ levels. Since the lowest ${ }^{7} F_{1}$ level lies at relatively high energy $\left(252 \mathrm{~cm}^{-1}\right)$, this process is not very likely below $60 \mathrm{~K}$. Because no obvious thermal line broadening could be observed, the increase of the diffusion constant cannot be explained by an increase of the spectral overlap.

The most probable explanation is that the temperature dependence is due to energy mismatch between the intrinsic $\mathrm{Eu}^{3+}$ ions. As a result of random strains and defects present to varying degrees in solids, not all of the intrinsic $\mathrm{Eu}^{3+}$ ions are in identical environments, so that inhomogeneous broadening of the energy levels and of optical transitions between these levels occurs. This energy mismatch can affect strongly the energy migration among the intrinsic $\mathrm{Eu}^{3+}$ ions. This is especially true at low temperatures where homogeneous linewidths are narrow and spectral overlap between neighbouring ions in a randomly strained system is in many cases negligible. Homogeneous linewidths of optical transitions in rare-earth ions at low temperatures are of the order of $\mathrm{MHz}$ $\left(\sim 10^{-4} \mathrm{~cm}^{-1}\right)$, whereas the observed linewidths of the ${ }^{7} F_{0} \leftrightharpoons{ }^{5} D_{0}$ transition in $\mathrm{Eu}^{3+}$ in $\mathrm{NaEuTiO}_{4}$ are of the order of $\mathrm{THz}$. So, the temperature dependence of the diffusion constant should be explained by the fact that the interactions between donor ions are phonon assisted.

The theory of phonon-assisted energy transfer was first developed by Orbach [21] and then worked out in detail by Miyakawa and Dexter [22], followed by Holstein et al. $[23,24]$. We have tried to fit the experimental data 
presented in fig. 12 to the general equation

$k_{\mathrm{H}}=A \operatorname{coth}(B / T)+C \exp (-\Delta / k T)+D T^{3}+E T^{7}$.

The first term represents the one-phonon assisted process. The second term shows the temperature dependence of the so-called Orbach process. The third and fourth term represent the two-site and the one-site Raman process.

The best fit is presented in fig. 12 and was obtained using the following equation:

$k_{\mathrm{H}}=A \operatorname{coth}(\Delta E / 2 k T)+B T^{7}$,

with $A=1.5 \times 10^{5} \mathrm{~s}^{-1} ; B=9.5 \times 10^{-4} \mathrm{~s}^{-1} \mathrm{~K}^{-7}$ and $\Delta E=2.3 \mathrm{~cm}^{-1}$. This implies that at low temperatures the one-phonon process dominates while at higher temperatures $(T>20 \mathrm{~K})$ the one-site Raman process become more important.

The probability of the direct process depends strongly on the so-called coherence factor $\left|\exp \left(\mathrm{i} \bar{q} \bar{r}_{i j}\right)-1\right|^{2}$ (ref. [25]). In order to calculate this coherence factor one needs to know the Debye temperature of the compound. We estimated $T_{\mathrm{D}}$ by giving it the value of the Debye temperature of $\mathrm{SrTiO}_{3}$, viz. $240 \mathrm{~K}$ (ref. [26]). For $r_{i j}=2 a$ the coherence factor becomes $\sim 1 \%$. This makes the direct process not very probable, but not impossible. The final value of the transfer rate depends on the product of the coherence factor and a prefactor. This factor depends on the interaction strength, the coupling of the ground states and the excited states with the phonons and other factors. Since it is extremely difficult even to estimate the prefactor, it is impossible to calculate the transfer rate.

One should realize that eq. (5) is a very rough estimation to describe the temperature dependence of the hopping rate. Figure 12 suggests that the one-phonon process occurs, but it is hard to prove. In fig. 2 we observe a deviation from the theoretical fit starting at $T \sim 35 \mathrm{~K}$. According to [24] the $T^{7}$ law is only valid for temperatures $T<T_{\mathrm{D}} / 7$. With $T_{\mathrm{D}}=240 \mathrm{~K}$ this would mean that $T<35 \mathrm{~K}$. We have no explanation for the higher-temperature behaviour.

Because the nature of the interaction is not known, we cannot prove that the energy migration at low temperatures is due to the linear crystal field. However, there is no doubt that energy migration among the Eu ${ }^{3+}$ ions in $\mathrm{NaEuTiO}_{4}$ takes place down to the lowest temperatures $(1.2 \mathrm{~K})$. Studies on similar systems are being performed.

\section{Acknowledgement}

This investigations were supported by the Netherlands Foundation for Chemical Research (SON) with financial aid from the Netherlands Organisation for Advancement of Pure Research (ZWO). 


\section{References}

[1] F. Kellendonk and G. Blasse, J. Chem. Phys. 75 (1981) 561.

[2] T. Förster, Ann. Phys. 2 (1948) 55.

[3] D.L. Dexter, J. Chem. Phys. 21 (1953) 836.

[4] Z.J. Kiss and H.A. Weakliem, Phys. Rev. Lett. 15 (1965) 457.

[5] W.C. Nieuwpoort and G. Blasse, Solid State Commun. 4 (1966) 379.

[6] B.G. Wybourne, J. Chem. Phys. 48 (1968) 2596.

[7] R.D. Peacock, Structure and Bonding 22 (1975) 83.

[8] B.R. Judd, Phys. Rev. 127 (1962) 750; J. Chem. Phys. 44 (1966) 839

[9] G. Blasse, J. Inorg. Nucl. Chem. 30 (1968) 656.

[10] G. Blasse and A. Bril, J. Chem. Phys. 48 (1968) 3652.

[11] C. Linarès and M. Blanchard, C.R. Acad. Sci. Paris 286B (1978) 387.

[12] G. Blasse and G.P.M. van den Heuvel, J. Solid State Chem. 10 (1974) 206.

[13] D.L. Huber, Phys. Rev. 20 (1979) 6, 2307.

[14] A.I. Burshtein, Sov. Phys. JETP 35 (1972) 882

[15] M. Inokuti and F. Hirayama, J. Chem. Phys. 43 (1965) 1978.

[16] R.K. Watts, in: Optical Properties of Ions in Solids, ed., B. Di Bartolo (Plenum Press, New York, 1975) p. 307.

[17] K.K. Ghosh and D.L. Huber, J. Lumin. 21 (1980) 225.

[18] W.J.C. Grant, Phys. Rev. B4 (1971) 648.

[19] J.P. van der Ziel, L. Kopf and L.G. van Uitert, Phys. Rev. B6 (1972) 615.

[20] G.E. Venikouas and R.C. Powell, J. Lumin. 16 (1978) 29.

[21] R. Orbach, in: Conf. Properties of Ions in Crystals (Wiley Interscience, New York, 1967) p. 445.

[22] T. Miyakawa and D.L. Dexter, Phys. Rev. B1 (1970) 2961.

[23] T. Holstein, S.K. Lyo and R. Orbach, Phys. Rev. Lett. 36 (1976) 15, 891.

[24] T. Holstein, S.K. Lyo and R. Orbach, in: Laser Spectroscopy of Solids, ed., W.M. Yen and P.M. Selzer, Topics in Applied Physics, Vol. 49 (Springer, Berlin, 1981) 39.

[25] T.F. Soules and C.B. Duke, Phys. Rev. B3 (1971) 262

[26] W.G. Stirling, J. Phys. C5 (1972) 2711. 